\title{
Multispecies aerosol evolution and deposition in a human respiratory tract cast model
}

\author{
Mahdi Asgari ${ }^{\text {a }}$, Francesco Lucci ${ }^{\text {a }}$, Arkadiusz K. Kuczaj ${ }^{\text {a,b, * }}$ \\ a PMI R\&D, Philip Morris Products S.A., Quai Jeanrenaud 5, CH-2000, Neuchâtel, Switzerland \\ ${ }^{\mathrm{b}}$ Multiscale Modeling \& Simulation, Dept. of Applied Mathematics, University of Twente, P.O.Box 217, 7500 AE, Enschede, the Netherlands
}

\section{A R T I C L E I N F O}

\section{Keywords:}

Human airways

Multispecies liquid aerosol dynamics

Condensation and evaporation

Hygroscopic growth

Computational fluid dynamics

\begin{abstract}
A B S T R A C T
Accurate predictions of aerosol transport, evolution, and deposition in the human airways are crucial for inhalation dosimetry investigations. Inhaled aerosol transported through the airways undergoes thermodynamic changes due to changes in temperature and humidity. Aerosol evolution processes are particularly important for liquid multispecies aerosols. These aerosols are sensitive to condensation and/or evaporation dynamics, which can modify gas/liquid partitioning of each species and particle size distribution. In this manuscript, we present computational fluid dynamics simulations of complex aerosol mixtures in a realistic geometry of the human respiratory tract cast model. We used a publicly available computational framework, AeroSolved, developed for simulation of evolving multispecies aerosol mixtures. We evaluated the dynamics of liquid particles in physiologically relevant conditions of $100 \%$ relative humidity at the temperature of $37^{\circ} \mathrm{C}$. We studied two separate inhalation flow scenarios: in the first case, a warm aerosol at $50^{\circ} \mathrm{C}$ was inhaled and subsequently cooled down while flowing in the airways. In the second case, an aerosol at room temperature was inhaled and heated up to the temperature of $37^{\circ} \mathrm{C}$. Our results demonstrated that aerosol evolution mainly occurs in the upper segments of the airways (throat and trachea) at the very short timescales. Apart from showing the significant influence of temperature and humidity conditions on aerosol dynamics and evolution, we also measured aerosol deposition fluxes investigating the dependence of the delivered aerosol mass on evolution mechanisms. We showed that the delivered regional mass of each species depends on the physicochemical properties of the mixture, and it is also significantly influenced by the airways' humidity and thermal conditions. It was also shown that the species-specific properties of the liquid mixture (e.g., activity coefficient) play an important role in gas/liquid partitioning of the species. With AeroSolved such dependencies can be further investigated with greater attention towards specific needs and physiologically important conditions (e.g., transient flow inhalation patterns, aerosol mixture composition and its properties).
\end{abstract}

\section{Introduction}

Our lungs are constantly exposed to aerosols from environmental or artificial sources. The delivered mass of aerosol to the human lung determines its consequent hazardous or therapeutic effects. Therefore, understanding aerosol dynamics is crucial for environmental and medical pulmonary dosimetry calculations (Heyder et al., 1986). The ultimate goal in dosimetry predictions is to provide

\footnotetext{
* Corresponding author. PMI R\&D, Philip Morris Products S.A., Quai Jeanrenaud 5, CH-2000, Neuchâtel, Switzerland.

E-mail address: Arkadiusz.Kuczaj@pmi.com (A.K. Kuczaj).
} 
accurate information on total, regional, and local aerosol deposition rates with respect to breathing conditions (Park \& Wexler, 2008; Stahlhofen et al., 1980). Evaluation of aerosol dynamics in vivo in a functional lung is challenging, if not impossible; therefore, physical in vitro (Asgari, Lucci, Bialek, et al., 2019; Asgari, Lucci, Kuczaj, et al, 2019; Grgic et al., 2004) and computational in silico (Frederix et al., 2018; Longest \& Xi, 2007) surrogate models have been developed and used to address scientific questions around this topic.

There have been various experimental and theoretical efforts to advance our understanding of aerosol processes. A variety of experimental techniques have been developed and reported in the literature to evaluate particles deposition in various segments of the respiratory tract using in vitro cast models exposures followed by analytical measurements (Nordlund et al., 2017) and imaging techniques (Dolovich, 2001; Fleming et al., 1996). Next to such experimental efforts, computational modeling has provided substantial insights into aerosol physical processes, also challenging the interpretation of experimental data (Longest \& Vinchurkar, 2007). Both approaches are crucial and required as complementary techniques. In addition, computational models validated with the available experimental data can be used as a reliable research tool in applications, where experiments are either not available or would require a substantial effort (Longest \& Hindle, 2010; Zhang et al., 2005).

Complex interactions of particle dynamics result in continuous aerosol evolution (aging) in terms of physical characteristics (particle size distribution) and chemical composition; this is particularly true in case of liquid aerosols, with regard to substancespecific mass transfer between the gas and liquid phases. In human lungs, the main physical mechanisms driving aerosol evolution are coalescence and condensation/evaporation (Hinds, 2012). Coalescence strongly depends on the amount and proximity of particles; thus, it becomes dominant only in case of large particle number densities (Lee \& Chen, 1984). Otherwise, this process is slow and occurs at a low magnitude. On the other hand, condensation and evaporation processes occur with time scales comparable to those of particles transport (Hinds, 2012). Condensation and evaporation change the local particle size distribution during inhalation and, thereby, significantly affect the transport and deposition of particles. Diffusion and sedimentation become important for very small and large particles respectively as complementing to their transport ability in particular when convection-dominated forces become less important (e.g., deep lungs aerosol dynamics).

Aerosols reaching the human airways might have a different temperature than that of the human body, resulting in a rapid change in their thermodynamical conditions. Respiratory flow induces pressure changes and subsequent evaporation of substances from the liquid phase; in contrast, increased humidity in the respiratory tract results in a reverse process of water condensation on the particles surface. Aerosol evolution triggered by flow, thermal, and humidity changes influences not only the physical characteristics of the particles but also the chemical composition of substances forming the aerosol. All these coupled processes would ultimately determine the local dosimetry of a multispecies aerosol in the human respiratory tract.

In this manuscript, we aim to address some of the above-mentioned open questions in understanding aerosol dynamics in the human airways by using detailed computational fluid dynamics simulations. To this end, we use a realistic geometry model of the human respiratory tract. We consider various thermal and humidity conditions of the airways and investigate the evolution of multispecies, relatively warm and cold aerosols flowing through the respiratory tract geometry. We evaluate the impact of aerosol evolution on regional aerosol deposition. This manuscript is divided into the following sections. Details on computational simulations, including computational mesh generation, model validation, applied boundary conditions, numerical schemes, and simulation cases are outlined in section 2 . The results of computational simulations for multispecies aerosols in the airways geometry are presented and discussed in section 3. Final remarks and conclusions are summarized in section 4.

\section{Materials and methods}

\subsection{Aerosol processes occurring during inhalation}

Aerosol transported through the human respiratory tract by diffusion and convection processes undergoes several physical processes before being delivered to the lungs. The transport trajectories of the particles differ from the ones of the carrier gas phase because of the particles drift induced by changes in flow direction, Brownian diffusion, gravity force, and drag forces acting on the particles. The mass exchange between the liquid and gas phases at the surface of the particles and the differences between species concentrations in the gas phase in the vicinity and at a distance from the surface of the particles result in the evolution and change in size of the particles through evaporation/condensation processes. For a single-species aerosol, condensation and evaporation are directly driven by the saturation conditions; in contrast, for multispecies aerosol, condensation might occur even in undersaturated conditions, depending on the fractions of species in the liquid and gas phases and the chemical interactions of these species in the mixture. For example, hygroscopic growth of particles will occur in the humidity conditions less than water saturated conditions (e.g., $90 \%$ relative humidity). For a liquid mixture, the subtle mass transfer at the gas-liquid interface will be modulated by the surrounding thermodynamical conditions. Considering inhalable particles smaller than a couple of micrometers, we assume limited possibility for the presence of Marangoni flows inside the particle; but, surface condensation/evaporation will certainly result in non-equilibrated chemical composition and species-specific diffusion-driven mixing of molecules inside the particle. Deposition of the particles on the surface is driven by Brownian diffusion, flow induced impaction and sedimentation. The relative contribution of these processes to the total deposited mass in various segments of the respiratory tract geometry depends on flow conditions, particles size, and complexity of the respiratory tract geometry in each segment.

In order to assess the aerosol processes contributing to aerosol evolution during inhalation, it is crucial to evaluate the time scales of these processes. We followed previously reported (Asgari, Lucci, Bialek, et al., 2019; Asgari, Lucci, Kuczaj, et al, 2019) formulations and procedure to calculate the time scales. In our simulations we considered an aerosol with the geometrical standard deviation (GSD) equal to $4 / 3$, and the particle number density of $10^{12} \mathrm{~m}^{-3}$. The choice here was arbitrary, neglecting coalescence and with the full 
understanding that it can be tuned towards specific needs of simulations. Here, we assumed a diluted and not very polydisperse aerosol, for which coalescence can be neglected. Condensation/evaporation, on the other hand, is a relatively rapid process occurring in time scales much smaller than the flow residence time (convection time scale) for the flow rate range of $1.5-30 \mathrm{~L} / \mathrm{min}$ considered in this manuscript. Regarding the total volume of the simulated geometry $(143 \mathrm{~mL})$, the estimated convection time scale for the simulated flow rates ranges from $0.286 \mathrm{~s}$ to $5.72 \mathrm{~s}$.

In this manuscript, we will address the modeling of these processes for inhaled aerosol using computational models developed for coupled aerosol processes such as drift and condensation/evaporation with transport of overall mass, momentum, and energy of the aerosol mixture. In the following sections, we introduce the computational simulation platform, and outline details of the geometry and mesh, validation of the models, boundary conditions, and numerical schemes.

\subsection{AeroSolved simulation platform}

Computational simulations were performed using the AeroSolved (AeroSolved, 2019) computational code. AeroSolved was developed to study aerosol dynamics starting from aerosol generation processes to aerosol evolution, transport, and deposition. Aerosol transport is calculated within an Eulerian-Eulerian representation, with particle size distribution modeled with the sectional and two-moment method (Frederix et al., 2016; Winkelmann et al., 2014). In this manuscript, we have used sectional method in cases, where we have simulated for a wide range of particles sizes and two-moment method in cases, where we have simulated a particle size distribution with specific particle mean diameter and geometric standard deviation. The code is implemented in the OpenFOAM open-source framework. AeroSolved solves the mass, momentum (Navier-Stokes), and energy conservation equations for the gas and liquid/solid (particle) phases in a mixture formulation. In the sectional method, particle size distribution is modeled with a discretized number of sections in the size space. For each size section, the transport equation of particle number density including the aerosol evolution source terms is solved. In the two-moment method, the shape of particle size distribution is fixed with log-normal distribution and a priori fixed width of the distribution (i.e., GSD). Therefore, only two degrees of freedom remain to be determined for the particle size distribution: particle number density, and mass mean diameter. The sectional method is capable of resolving details of the particle size distribution; but, for the same reason, it is computationally more demanding than the two-moment method (see (AeroSolved, 2019)). Here, we have mainly mentioned the important parts of the model from the perspective of the aerosol evolution namely the condensation/evaporation source term in the multispecies context.

For the condensation/evaporation model, the species-specific $j$ evaporation rate $I_{j}$ follows the formulation proposed by (Newbold \& Amundson, 1973) and it is calculated as:

$$
I_{j}=\frac{2 \pi d D_{j} \rho_{j}^{g}}{p} f\left(\zeta_{j}\right)\left[\exp \left(\zeta_{j}\right) p_{j}-p_{j}^{\text {surf }}\right], j=1 \ldots N
$$

where $\rho_{j}^{g}$ is the species gas density, $p$ the total pressure, $p_{j}$ the vapor pressure of species $j$ far away from the particle, $p_{j}^{\text {surf }}$ the vapor pressure at the particle surface, $D_{j}$ the gas diffusivity of the species $j$, and $N$ the total number of phase changing species in the aerosol mixture. The function $f\left(\zeta_{j}\right)$ introduces a modification of the standard single species condensation rate:

$$
\begin{aligned}
& f\left(\zeta_{j}\right)=\frac{\zeta_{j}}{1-\exp \left(\zeta_{j}\right)}, \\
& \zeta_{j}=\frac{D_{I}}{D_{j}} \log \left[\frac{p-\sum_{k=1}^{N} p_{k}^{\text {surf }}}{p-\sum_{k=1}^{N} p_{k}}\right],
\end{aligned}
$$

with $D_{I}$ as the carrier gas diffusivity. The vapor pressure of species $j$ far away from the particle, $p_{j}$, is calculated with the partial pressure relation $x_{j}=\frac{p_{j}}{p}$ with $x_{j}$ being the vapor mole fraction with respect to the gas mixture. For species-specific vapor pressure calculations at the particle surface, we apply the generalized Raoult's law for the mixture with activity coefficients introduced to account for non-ideal multispecies mixture behavior:

$$
p_{j}^{\text {surf }}=\gamma_{j} w_{j} p_{j}^{\text {sat }}
$$

where $w_{j}$ is the liquid mole fraction with respect to the mixture, $p_{j}^{\text {sat }}$ is the saturation vapor pressure, and $\gamma_{j}$ is the activity coefficient accounting for the non-ideal behavior of the species in the mixture.

\subsection{Geometry and mesh}

Computational simulations were conducted in a human respiratory tract geometry with up to six generations of the tracheobronchial tree, originally obtained and introduced by Zhang et al. (Zhang et al., 2012). The geometry of the respiratory tract was divided into four segments: the throat, trachea, generations 1-3, and generations 4-6 of the tracheobronchial tree. This segmentation was made to analyze the regional deposited aerosol mass in our computational domain. Table 1 indicates the internal surface area of each geometry segment. 
Computational meshes of varying density were generated with 6,10 , and 14 million computational mesh cells for assessing numerical accuracy. The mesh with 6 million cells was generated as the starting point for the computational investigations with a first layer thickness of $50 \mu \mathrm{m}$ in the boundary layer and a base cell size of $360 \mu \mathrm{m}$ in the domain. Mesh sensitivity analysis was performed, and the error for the total value of the mass deposition flux on the geometry surface for meshes composed of 10 and 14 million cells was less than $5 \%$. Taking this into account we concluded to perform our simulations and analysis using the mesh with 10 million cells.

\subsection{Verification of the condensation/evaporation model}

We compared our results on the total deposition fraction in the full respiratory tract geometry with those of Lagrangian modeling presented by Zhang et al. (Zhang et al., 2012). Fig. 2 (a) shows the deposition fraction calculated for a particle diameter range of $100 \mathrm{~nm}-40 \mu \mathrm{m}$.

For verifying the condensation/evaporation model, we have used experimental data (Tu \& Ray, 2005) from an experiment with a single glycerol particle suspended with an electrodynamic balance inside a chamber, into which a salt solution is introduced at the initial time. As a direct result, water vapor starts rising from the solution and condenses on the particle surface. The relative humidity in the chamber and size growth of the particle are measured and reported. In order to reproduce the experimental conditions, water vapor mass fraction values corresponding to the measured relative humidity over time were imposed at the boundaries of the simulation domain. Activity coefficients of aqueous glycerol solutions were implemented by using the correlations obtained in (Tu \& Ray, 2005). The particle size growth measured in our simulation was in agreement with the experimental measurements, as shown in Fig. 2(b). It was possible to reach this excellent agreement by appropriate treatment of the non-ideality of water-glycerol mixture by considering a model for the activity coefficient. Assuming a constant activity coefficient of one, which neglects the non-ideal behavior of the water-glycerol mixture resulted in an underestimation of the condensation rate of about $30 \%$, showing the importance of an appropriate model for the activity coefficient (Asgari, Lucci, Bialek, et al., 2019; Asgari, Lucci, Kuczaj, et al, 2019).

\subsection{Boundary conditions and numerical schemes}

The boundary conditions used in the simulations are summarized in Table 2. We assumed the aerosol gas and liquid mixture at the inlet to be at equilibrium for no-flow conditions and imposed a saturation boundary condition for the gas phase of different species at the inlet. The imposed saturation condition at the inlet for gas mass fractions were calculated with reference to the temperature dependent saturation vapor pressure (See Appendix A), Raoult's law and molecular mass of the simulated species:

$$
\begin{gathered}
x_{j}^{\text {sat }}=\gamma_{j} w_{j} p_{j}^{\text {sat }} / p, \\
Y_{j}^{\text {sat }}=\frac{x_{j}^{\text {sat }} M_{j}}{\sum_{j} x_{j}^{\text {sat }} M_{j}},
\end{gathered}
$$

in which $x_{j}^{\text {sat }}$ and $Y_{j}^{\text {sat }}$ stand for the saturated mole and mass fractions in the gas phase, and $M_{j}$ is molecular mass of the species $j$. It should be noted that the imposed saturation condition does not consider the curvature dependent vapor pressure at particles surface, known as Kelvin effect. Therefore, the considered equilibrium condition is not particle size dependent and does not depend on particle size distribution at the inlet.

At the geometry walls, we imposed saturated conditions for water vapor, representing the humidity of human lungs. We considered a relative humidity of $100 \%$ at the walls, which meant that the water vapor pressure was equal to the saturation vapor pressure at the temperature of the walls. We imposed the corresponding gas mass fraction of water on the walls. For the rest of the species, we imposed a zero-gradient boundary condition at the walls. A zero-gradient boundary condition at the walls implies no flux and, therefore, no absorption of the gas phase through the walls. For the particle number density at the walls, we assumed a perfectly absorbing surface with zero concentration of particles at the walls to calculate the gradient of particle concentration leading to diffusional deposition. The details of the boundary condition for the particle deposition have been previously explained in (Frederix et al., 2017). The particle size distribution at the inlet was assumed to follow a log-normal distribution shape with fixed particle size distribution characteristics of mass mean diameter and geometric standard deviation (GSD).

The steady-state simulations were initialized with a fully developed flow (velocity and pressure fields in the computational domain) by using the single-phase simulation results. This initialization step enabled faster convergence of the simulations in case of multispecies/multiphase mixtures. However, it must be noted that the velocity and pressure fields were not frozen in order to allow them to

Table 1

Internal surface area of the respiratory tract geometry segments as shown in Fig. 1.

\begin{tabular}{ll}
\hline Segments & Surface area $\left(\mathrm{cm}^{2}\right)$ \\
\hline Throat & 124 \\
Trachea & 56 \\
G1-3 & 87 \\
G4-6 & 234 \\
\hline
\end{tabular}




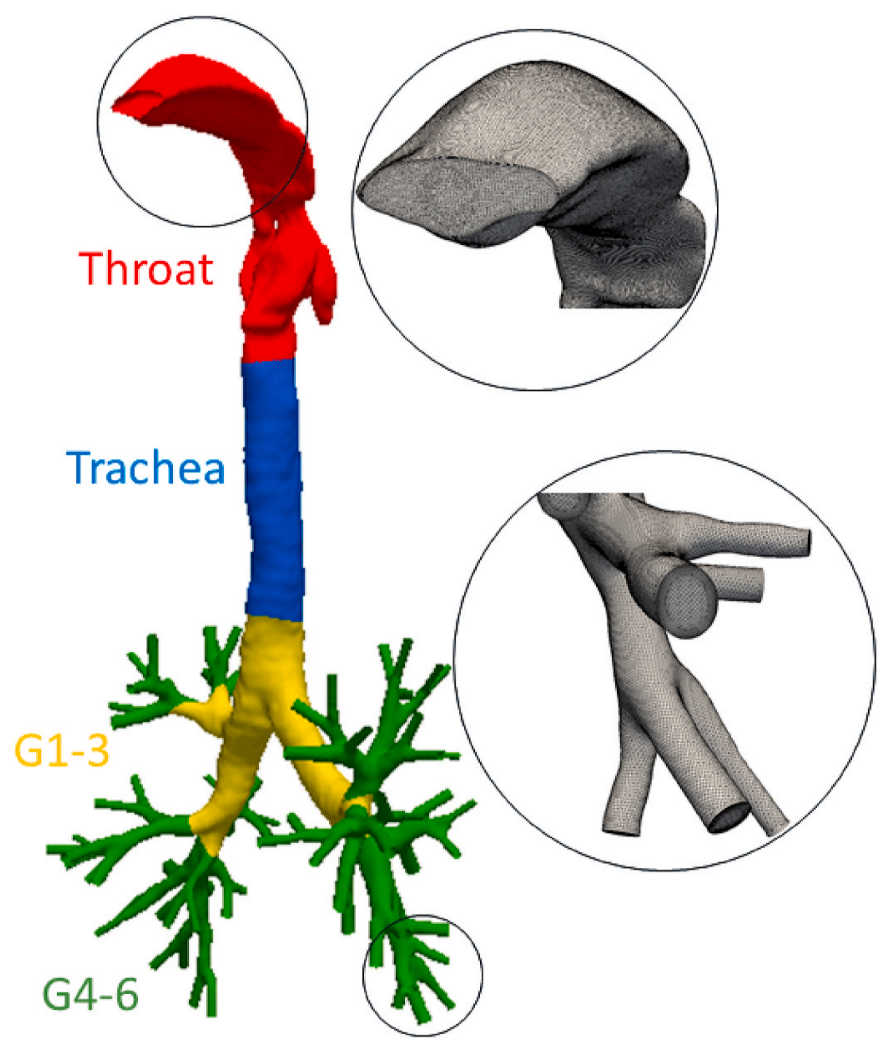

Fig. 1. Geometry and computational mesh of the human respiratory tract up to six generations of the tracheabronchial tree.

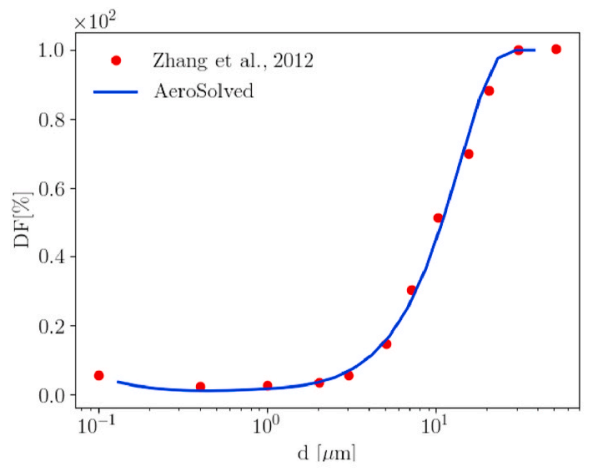

(a)

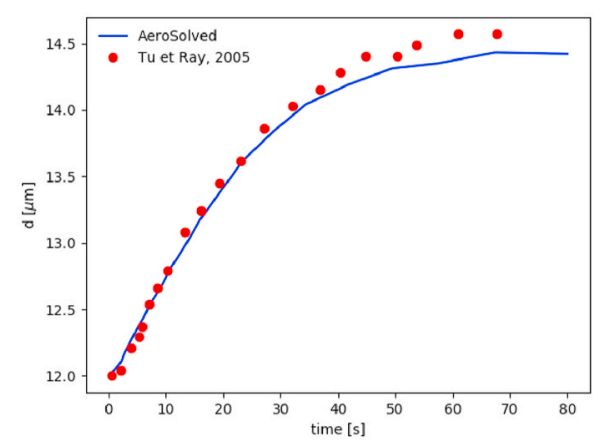

(b)

Fig. 2. (a) Deposition fraction (DF [\%]) versus particle diameter compared with the results of Lagrangian simulations taken from Zhang et al. (Zhang et al., 2012) performed for the respiratory tract geometry shown in Fig. 1. (b) Diameter of single glycerol particle evolution in time. The simulation results were compared with the experimental data from (Tu \& Ray, 2005).

change according to the thermal effects and temperature dependent physical properties of the mixture. Time integration was performed with a first-order Euler implicit scheme. Discretization of divergence terms for velocity, temperature, and pressure was performed with a second-order upwind scheme. Divergence terms in the transport equations for the gas mass fraction, liquid mass fraction, and particle size distribution were discretized by using a second-order van Leer limiter scheme (Van Leer, 1979). All gradient and Laplacian terms were discretized with a second-order accuracy central scheme.

\subsection{Simulation cases and conditions}

Various simulation cases were considered to investigate the evolution and deposition of aerosol in the human respiratory tract geometry depending on the aerosol mixture composition, flow rates, thermal and humidity conditions, and inlet particle size 
Table 2

Description of boundary conditions (BCs) for various flow fields.

\begin{tabular}{llll}
\hline Field/property & inlet & outlets & walls \\
\hline Pressure & Zero-gradient & Fixed value & Zero-gradient \\
Temperature & Fixed value & Zero-gradient & Fixed value \\
Velocity (flow) & Fixed value & Zero-gradient & No-slip \\
Velocity (particles) & Fixed value & Zero-gradient & Deposition velocity \\
Mass fractions (liquid) & Fixed value & Zero-gradient & Deposition BC \\
Mass fractions (gas) & Saturated conditions & Zero-gradient & Zero-gradient (for water saturated conditions) \\
Particle number density & Log-normal distribution & Zero-gradient & Deposition BC \\
\hline
\end{tabular}

distribution. For an overview, see a collection of all cases in Table 3 with the inlet mass fractions in liquid and gas phases (calculated using the saturation boundary condition as explained in section 2.5) summarized in Table 4.

For the chemical composition we have chosen liquids present in the electronic cigarettes (the so-called e-liquids), which are mostly composed of glycerol, propylene glycol, water, and additional species like nicotine, and a variety of flavors. Such choice, apart from the practical importance, gives us a possibility to consider mixtures with a large span of their varying properties (e.g. boiling temperatures, viscosity, heat of evaporation). These properties are relevant both for the aerosol generation and evolution processes.

Simulations representative of non-evolving aerosol were conducted for pure glycerol (VG) aerosol particles (case A). VG has low vapor pressure values in the considered temperature range of $25-50^{\circ} \mathrm{C}$; therefore, one can assume that particles composed of pure glycerol do not significantly evolve in size under thermal effects (without humidity effects) while being transported through the respiratory tract geometry. With our computational simulations, we have verified the above-stated assumption that glycerol particles do not evolve in the considered range of temperatures (Asgari, Lucci, Bialek, et al., 2019; Asgari, Lucci, Kuczaj, et al, 2019). In these non-evolving simulations, in order to capture the full range of particle sizes with a single simulation a sectional model was used (see Section 2.2). The range of particle mean diameters considered in our non-evolving simulations was $50 \mathrm{~nm}-50 \mu \mathrm{m}$.

Simulations representative of evolving aerosol were conducted for aerosols containing volatile species such as propylene glycol (PG), water, and nicotine. For an evolving aerosol with temperature and pressure dependent physical properties, thermal and humidity conditions trigger particle size evolution in the respiratory tract. The evolving aerosols were simulated with a selected particle size distribution (log-normal distribution with selected particle mean diameter and geometric standard deviation, GSD, of 4/ 3) using the two-moment aerosol model. For multispecies evolving aerosol simulations, in order to represent the humidity released from the respiratory tract in the airways, we imposed a saturated boundary condition for water in the gas phase at the walls, keeping a relative humidity of $100 \%$ in the region adjacent to the walls. VG is a hydrophilic species with the ability to absorb water from the gas phase. In order to take into account such hygroscopic properties of VG, we modified the vapor pressure with the activity coefficients reported in the literature (Tu \& Ray, 2005) for this species. For multispecies aerosols containing nicotine, we considered the non-ideal vapor pressure behavior of nicotine in the presence of water by applying nicotine activity coefficients in the condensation/evaporation model. There is limited information on the values of nicotine activity coefficients in simulated aerosol compositions. Therefore, we simulated aerosol evolution considering a range of activity coefficients reported for nicotine in aqueous solutions (from 1 to 60) (Banyasz, 1999).

We compared the results for evolving aerosol with those obtained with exactly the same simulation conditions and material properties, but without the possibility for the aerosol evolution (cases B-G). In the cases simulating non-evolving aerosols, the corresponding phase-related mass transfer terms were canceled in the simulations, which allowed us to directly compare simulations performed this way with the ones in which species mass transfer was present. For these simulations, we selected propylene glycol as being prone to evaporation and significantly impacted by the thermodynamical conditions in the considered temperature and pressure ranges.

For the thermal conditions, we considered two distinct temperature scenarios between 25 and $50^{\circ} \mathrm{C}$ with respect to the human body

Table 3

Simulation cases coded for various compositions, inlet temperatures $\left(T_{\text {inlet }}\right)$, inhalation flow rate $(\mathrm{Q})$, relative humidity $(\mathrm{RH})$, and particles mass mean diameters at the inlet.

\begin{tabular}{|c|c|c|c|c|c|c|}
\hline Code & Evolving/Non-evolving & Composition & $T_{\text {inlet }}\left[{ }^{\circ} \mathrm{C}\right]$ & $\mathrm{Q}[\mathrm{L} / \mathrm{min}]$ & RH [\%] & $\bar{d}_{\text {inlet }}[\mu m]$ \\
\hline A & Non-evolving & Air-VG & 50 & $1.5,15,30$ & - & $0.05-50$ \\
\hline B & Evolving & Air-PG & 50 & $1.5,30$ & - & 1 \\
\hline $\mathrm{C}$ & Non-evolving & Air-PG & 50 & 1.5 & - & 1 \\
\hline $\mathrm{D}$ & Evolving & Air-PG & 50 & 1.5 & - & 4 \\
\hline $\mathrm{E}$ & Non-evolving & Air-PG & 50 & 1.5 & - & 4 \\
\hline $\mathrm{F}$ & Evolving & Air-PG & 50 & 1.5 & - & 0.4 \\
\hline G & Non-evolving & Air-PG & 50 & 1.5 & - & 0.4 \\
\hline $\mathrm{H}$ & Evolving & Air-PG & 25 & 30 & - & 1 \\
\hline I & Non-evolving & Air-PG & 25 & 30 & - & 1 \\
\hline $\mathrm{J}$ & Evolving & Air-VG-PG-Water & 50 & 1.5 & 100 & 1 \\
\hline $\mathrm{K}$ & Non-evolving & Air-VG-PG-Water & 50 & 1.5 & 100 & 1 \\
\hline $\mathrm{L}$ & Evolving & Air-VG-PG-Water-Nicotine & 50 & 1.5 & 100 & 1 \\
\hline
\end{tabular}


Table 4

Species mass fractions in liquid (Z) and gas (Y) phases at the inlet boundary condition.

\begin{tabular}{|c|c|c|c|c|c|c|c|c|c|c|}
\hline Code & $Z_{\text {Air }}[-]$ & $Z_{\text {Water }}[-]$ & $Z_{P G}[-]$ & $Z_{V G}[-]$ & $Z_{\text {Nicotine }}[-]$ & $Y_{\text {Air }}[-]$ & $Y_{\text {Water }}[-]$ & $Y_{P G}[-]$ & $Y_{V G}[-]$ & $Y_{\text {Nicotine }}[-]$ \\
\hline A & 0 & 0 & 0 & $1 \cdot 10^{-3}$ & 0 & $99.9 \cdot 10^{-2}$ & 0 & 0 & 0 & 0 \\
\hline B-G & 0 & 0 & $1 \cdot 10^{-3}$ & 0 & 0 & $99.55 \cdot 10^{-2}$ & 0 & $3.5 \cdot 10^{-3}$ & 0 & 0 \\
\hline $\mathrm{H}, \mathrm{I}$ & 0 & 0 & $1 \cdot 10^{-3}$ & 0 & 0 & $99.86 \cdot 10^{-2}$ & 0 & $4 \cdot 10^{-4}$ & 0 & 0 \\
\hline $\mathrm{J}, \mathrm{K}$ & 0 & $2 \cdot 10^{-4}$ & $4 \cdot 10^{-4}$ & $4 \cdot 10^{-4}$ & 0 & $91.85 \cdot 10^{-2}$ & $7.9 \cdot 10^{-2}$ & $1.5 \cdot 10^{-3}$ & $1.2 \cdot 10^{-5}$ & 0 \\
\hline $\mathrm{L}$ & 0 & $1.8 \cdot 10^{-4}$ & $4 \cdot 10^{-4}$ & $4 \cdot 10^{-4}$ & $2 \cdot 10^{-5}$ & $92.42 \cdot 10^{-2}$ & $7.1 \cdot 10^{-2}$ & $1.55 \cdot 10^{-3}$ & $1.2 \cdot 10^{-5}$ & $2.3 \cdot 10^{-3}$ \\
\hline
\end{tabular}

temperature $37^{\circ} \mathrm{C}$. These two temperatures correspond to potential aerosol generation using nebulization at the ambient (room temperature) conditions and thermal aerosolization due to nucleation of supersaturated vapors as for the electronic cigarettes. We concentrated on the assessment of evaporation/condensation processes considering diluted aerosol concentration for the inlet conditions to avoid additional intricate interactions with coagulation processes occurring at large particle number densities. This choice was arbitrary as AeroSolved is capable to include Brownian coagulation (Lee \& Chen, 1984).

Inhalation flow rates depend on breathing conditions, which may vary among people and respiration modes (e.g., rest, exercise, and intense exercise). Here, we have simulated cases with flow rates of $1.5,15$, and $30 \mathrm{~L} / \mathrm{min}$, which are representative for inhalation flow rates in various conditions.

\section{Results and discussion}

Here we present the results of computational simulations for a variety of simulations cases introduced in section 2.6. We started with evaluation of non-evolving aerosol (section 3.1), then considering evolving single-species (sections 3.2.1 and 3.2.2) and multispecies mixtures (section 3.2.3). Finally, the last section of the results presents multispecies aerosol simulations including small mass fraction of nicotine in the mixture (3.2.4). The scientific intention behind this investigation was to investigate the dynamics of aerosol in the presence of nicotine with highly non-ideal vapor pressure behavior. In particular, we were interested in gas/liquid partitioning of nicotine due to aerosol evolution.

\subsection{Non-evolving aerosol simulations}

First, we looked into transport and deposition of non-evolving VG particles. Particle deposition was evaluated by calculating the deposition fraction (DF) as defined in Equation (7). The regional deposition fraction of particles for a large range of particle mean diameters (50nm to $50 \mu \mathrm{m}$ ) was calculated (Fig. 3). Deposition fraction was defined as the ratio of the flux of particles 'depositing on the walls' in each segment of the respiratory geometry divided by the flux of particles 'entering the respiratory tract through the inlet'.

$$
D F=\frac{\sum_{F \in \text { Wall }} \Phi_{F}}{\sum_{F \in \text { Inlet }} \Phi_{F}} \times 100,
$$

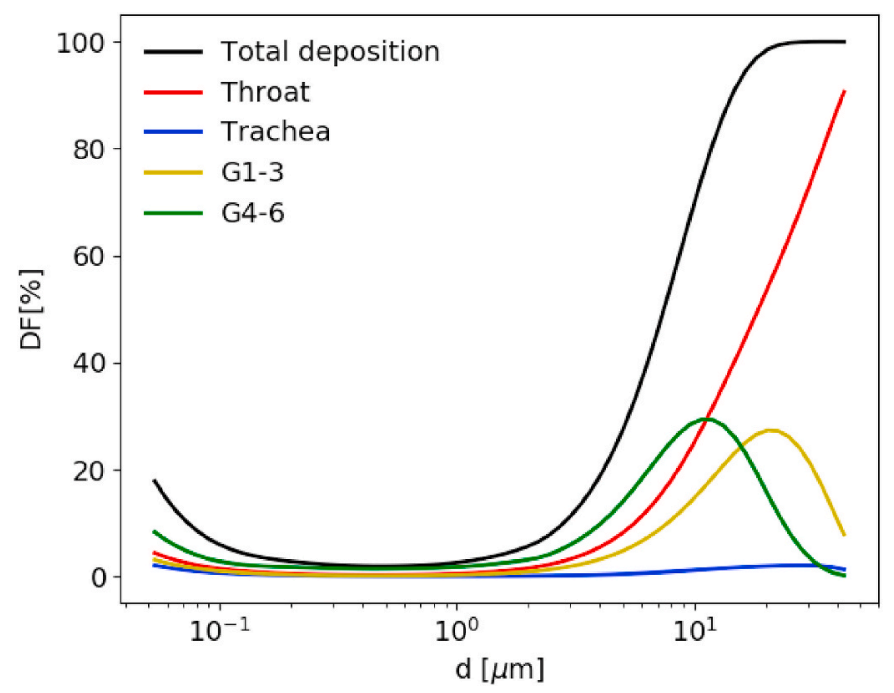

Fig. 3. Regional deposition fraction percentage of non-evolving VG particles versus particle mass mean diameter for steady inhalation flow rate of $1.5 \mathrm{~L} / \mathrm{min}$. 
where $\Phi_{F}$ is the total flux of particles with specific diameter size crossing computational cell surface $F$ on the wall or inlet. The simulated flow rate was set at $1.5 \mathrm{~L} / \mathrm{min}$, representative of aerosol inhalation flow rate.

The deposition fraction of sub-micrometer particles $<1 \mu \mathrm{m}$ is less than $20 \%$ in all segments of the upper respiratory tract geometry, with the maximum deposition fraction occurring at deeper airway generations (generations four to six in the geometry). Particle deposition in the sub-micrometer diameter range is governed by Brownian diffusion mechanism and, correlates with the surface area of the geometry walls in various airways segments (Table 1). Branching of the airways increases the exposed surface area and decreases the branch diameter. Consequently, this enhances the diffusion driven particle deposition on the surfaces. On the other hand, the dominant deposition mechanism for particles larger than $1 \mu \mathrm{m}$ in diameter are impaction and sedimentation. The maximum deposition fraction for this range of particle diameter occurs in the throat region and bifurcations of the geometry. Filtration of particles by inertial deposition in the upper respiratory tract does not allow particles delivery to the lower respiratory tract regions. These results are consistent with both experimental and numerical findings obtained by other researchers (Zhang et al., 2012).

\subsubsection{Flow rate dependency on regional particles deposition}

In this section we investigated the influence of inhalation flow rates on the regional deposition fraction. Fig. 4 (a) shows the total deposition fraction at different flow rates, while Fig. 4 (b) shows the regional deposition fraction in the throat and generations 4 to 6 of the tracheobronchial tree. Total deposition fraction curve is shifted towards smaller particles for increased flow rates. For regional deposition, it is seen that the deposition fraction of sub-micrometer particles diminishes with increased flow rates. Inertial deposition is enhanced by increased flow rate, and therefore, filtration of large particles in the throat region is increased. At the highest flow rate of $30 \mathrm{~L} / \mathrm{min}$ almost all particles larger than $10 \mu \mathrm{m}$ are deposited in the throat and very few are available to deposit in generations higher than 3. On the other hand, at $1.5 \mathrm{~L} / \mathrm{min}$, a smaller fraction of particles with $d>10 \mu \mathrm{m}$ deposits in the throat and, therefore, a more significant fraction reaches and deposits in higher generations (see Fig. 4 (b)). By performing these simulations, we obtained relevant insights into the flow-related dependencies of particle deposition for non-evolving aerosols.

\subsection{Evolving aerosol simulations}

We conducted aerosol flow simulations including volatile species such as PG and water. We compared the results of evolving aerosol deposition with those obtained with the assumption of no evolution in the computational simulations.

\subsubsection{Single-species aerosol evolution and deposition}

First, we conducted evolving aerosol simulations for a single-species semi-volatile PG aerosol diluted in air. The simulations were conducted for a warm mixture of aerosol at $50^{\circ} \mathrm{C}$, flowing through the respiratory tract geometry at a fixed wall temperature of $37^{\circ} \mathrm{C}$. The mass mean diameter of particle size distribution at the inlet was set at $1 \mu \mathrm{m}$ to investigate the sensitivity of deposition mechanisms to particle size in the vicinity of dominance of both diffusional and inertial deposition regimes. This case was labeled with code B in Tables 3 and 4, where main simulation parameters are listed. Fig. 5 presents two snapshots of the geometry, with visualized fields of the condensation rate (a) and mass mean diameter of particles (b). These snapshots show the origin and result of particle size evolution in the airways geometry. The major condensation zone appears in proximity to the inlet region, where the warm inhaled vapor at $50^{\circ} \mathrm{C}$ is mixed with stationary air at a temperature $37^{\circ} \mathrm{C}$ because of the selected boundary conditions. The second zone of condensation happens adjacent to the throat upper wall, where the inflow jet reaches the wall. The recirculation of air flow in the throat extends the condensation zone to the internal domain inside the mouth cavity. The temperature decrease of the aerosol at the inlet from $50^{\circ} \mathrm{C}$ to $37^{\circ} \mathrm{C}$ in the respiratory tract geometry triggers particle size growth and increases the mass mean diameter of the particles from $1 \mu$ mat the inlet to a maximum value of $1.54 \mu \mathrm{m}$ (see Fig. 5 (b)). It can be observed that the size evolution of particles is restricted to the mouth and throat regions, where the inhaled aerosol equilibrates with the thermal conditions of the respiratory flow.

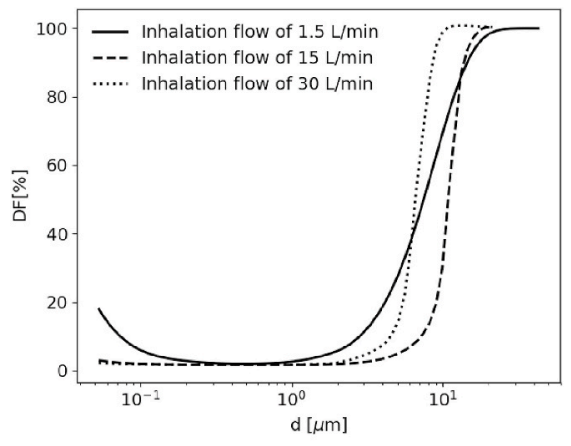

(a)

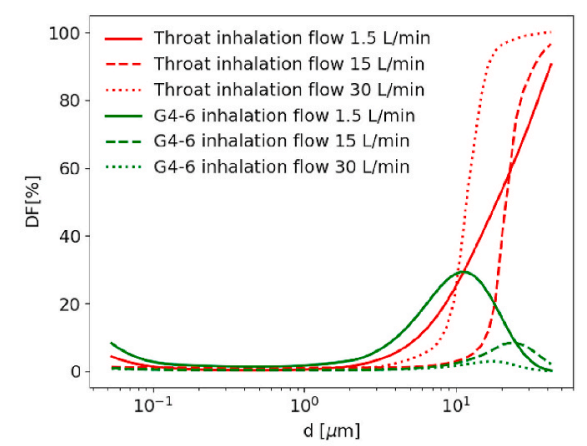

(b)

Fig. 4. (a) Total deposition fraction in the respiratory tract geometry compared for various flow rates representative of steady inhalation flow rates of $1.5,15$, and $30 \mathrm{~L} / \mathrm{min}$. (b) Regional deposition fraction calculated in throat and generations four to six of tracheobronchial tree compared for various flow rates. 
Fig. 6 shows a streamline of the flow in the geometry and the increase in the mass mean diameter of the particles along this line for two distinct flow rates of 1.5 and $30 \mathrm{~L} / \mathrm{min}$. In case of the $1.5 \mathrm{~L} / \mathrm{min}$ inhalation flow rate, the size growth of particles in the mouth cavity ( $<5 \mathrm{~cm}$ along the streamline) results in a mass mean diameter of $1.25 \mu \mathrm{m}$, which means a $25 \%$ increase with respect to the inlet diameter of particles at the inlet. Following the streamline in the throat region ( $<10 \mathrm{~cm}$ along the streamline), particles grow further to a mass mean diameter of $1.52 \mu \mathrm{m}$. Particles size does not increase in the deeper segments of the respiratory tract including the trachea and generations of the bronchial tree.

Different inhalation flow rates will change local thermokinetics conditions. To quantify the sensitivity of particle size evolution to inhalation flow rate, we compared the size evolution of particles along the flow streamlines for two flow rates of $1.5 \mathrm{and} 30 \mathrm{~L} / \mathrm{min}$. The other conditions including, temperature, inlet particle size, and boundary conditions were kept exactly the same for the two simulations. It can be seen in Fig. 6 that at the increased flow rate of $30 \mathrm{~L} / \mathrm{min}$, particles grow only to a mass mean diameter of $1.2 \mu \mathrm{m}$ in the throat region compared to $1.5 \mu \mathrm{m}$ at the lower flow rate. However, starting from the trachea region ( $>20 \mathrm{~cm}$ along the streamline), particles reach the same particle diameter of about $1.5 \mu \mathrm{m}$. Therefore, under the present assumptions, we observed that for increased inhalation flow rate, particles evolution occurs in deeper segments of the respiratory tract where, however, the same equilibrium size of particles is reached.

Next, we evaluated the influence of aerosol evolution on regional particle deposition. Fig. 7 shows the deposition fluxes of PG aerosol calculated in different segments of the respiratory tract geometry and compares the deposition fluxes for evolving and nonevolving simulations conducted with the same material properties, inlet aerosol mixture, and particles mass mean diameter at the inlet. Therefore, the only difference between the simulation cases was the inclusion of the condensation/evaporation process model in case of evolving aerosol simulations (cases B and C in Table 3). Fig. 7 (a) shows the particle number deposition for evolving and nonevolving cases in all segments of the geometry. For evolving aerosol, increased particle number deposition flux results from inertial and gravitational sedimentation deposition of the size-grown particles due to condensation. Fig. 7 (b) shows the comparison of regional deposited mass flux between the two cases and indicates increased deposited mass due to particle evolution in case of evolving aerosol. The increase in deposited mass results from not only the larger number of depositing particles (i.e., larger deposition fraction) but also the larger delivered mass of individual depositing particles.

Fig. 8 shows a similar analysis of the regional deposited mass for various inlet particle mass mean diameters of $400 \mathrm{~nm}, 1 \mu \mathrm{m}$, and $4 \mu \mathrm{m}$. The GSD of the particle size distribution for all three cases was set to $4 / 3$ at the inlet. The changes in deposition between evolving and non-evolving aerosols are related and consistent in terms of increase in the deposition of large particles relative to small particles (e.g., $4 \mu \mathrm{m}$ compared with $1 \mu \mathrm{m}$ ). Therefore, increased deposition of evolving aerosol can be attributed to the particle size growth and their subsequent increased inertial and gravitational sedimentation deposition.

\subsubsection{Aerosol evolution and deposition under evaporating thermal conditions}

In this section, we simulated an evolving aerosol with a relatively cold inlet temperature of $25^{\circ} \mathrm{C}$ entering the respiratory tract geometry with a warmer set temperature of $37^{\circ} \mathrm{C}$ at the walls (case $\mathrm{H}$ in Table 3). Fig. 9 (a) shows the aerosol liquid mass fraction. The liquid mass fraction decreases with the flow of the aerosol through the respiratory tract geometry, indicating particles evaporation with dominant mass transfer from the liquid to gas phase. The considered flow rate of $30 \mathrm{~L} / \mathrm{min}$ was constrained with the inlet diameter, which resulted in a jet flow through the inlet. The inhalation jet hits the walls in the throat and breaks into secondary flows. Consequently, the flow is heated up by the wall at $37^{\circ} \mathrm{C}$ resulting in evaporation and subsequently smaller particle sizes. Fig. 9 (b) shows the mass mean diameter of the particles and its changes through the geometry due to particles evaporation. Comparing these
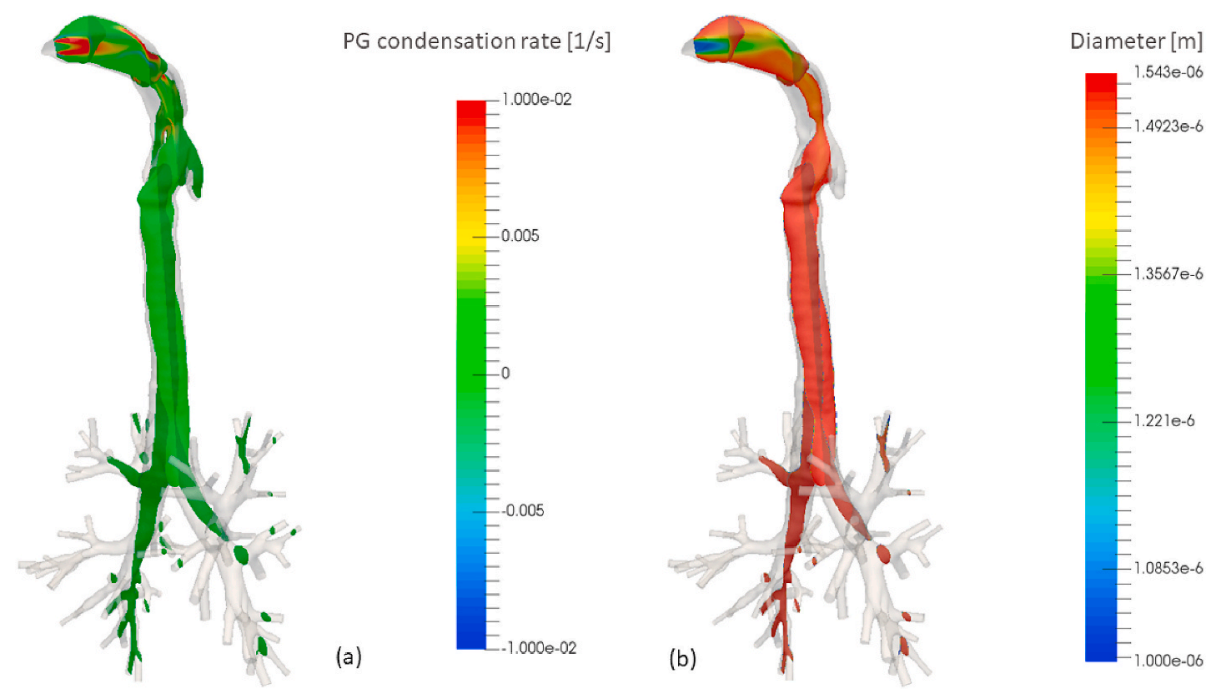

Fig. 5. (a) Snapshot of the condensation rate of a single species PG aerosol due to the change in temperature from the inlet at $50^{\circ} \mathrm{C}$ to the body temperature at $37^{\circ} \mathrm{C}$. (b) Snapshot of the mass mean diameter of the particles flowing through the respiratory tract geometry. 


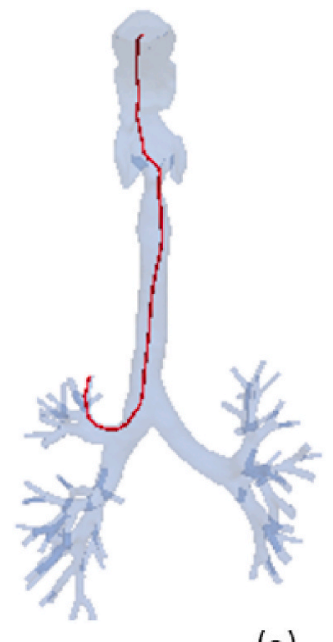

(a)

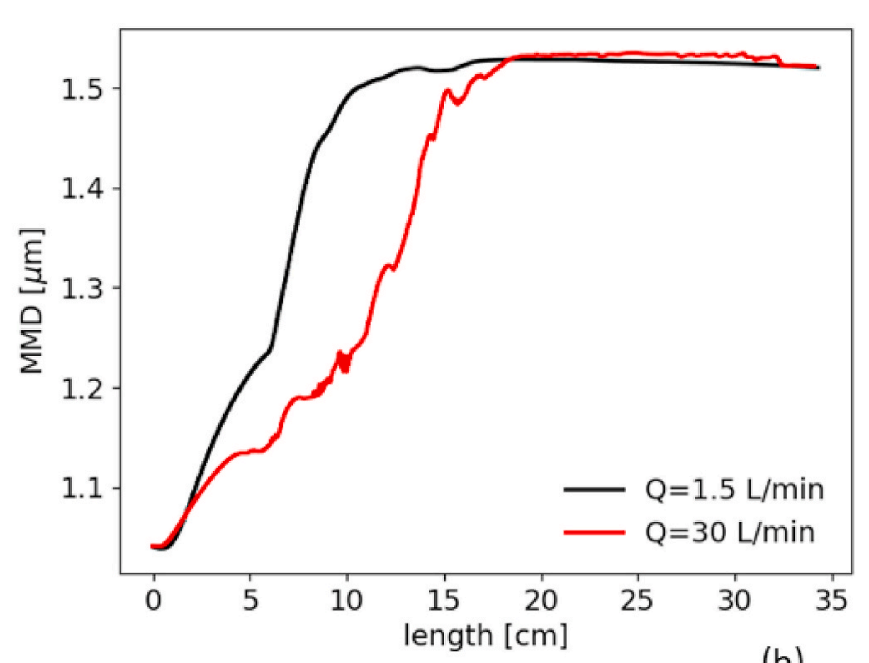

(b)

Fig. 6. (a) A streamline of the flow along the respiratory tract geometry. (b) Particles mass mean diameter versus streamline length for two inhalation flow rates of 1.5 and $30 \mathrm{~L} / \mathrm{min}$.

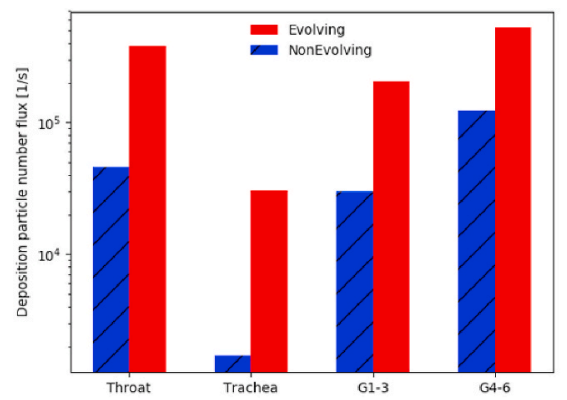

(a)

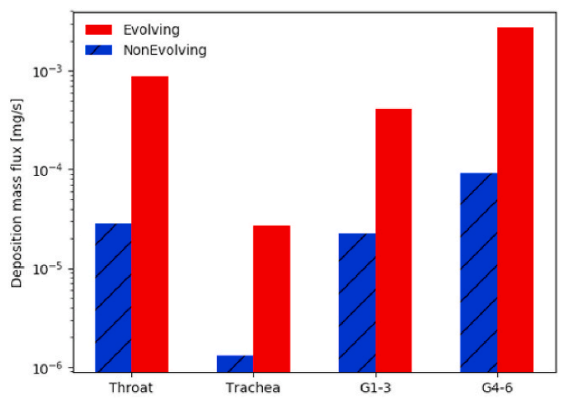

(b)

Fig. 7. (a) Particle number flux deposition in various segments of the cast geometry as labeled in Fig. 1 and compared for non-evolving and evolving aerosol simulations with the same inlet mixture and thermal properties (simulation cases B and C in Table 3). (b) Regional mass deposition flux compared for non-evolving and evolving aerosol simulations.

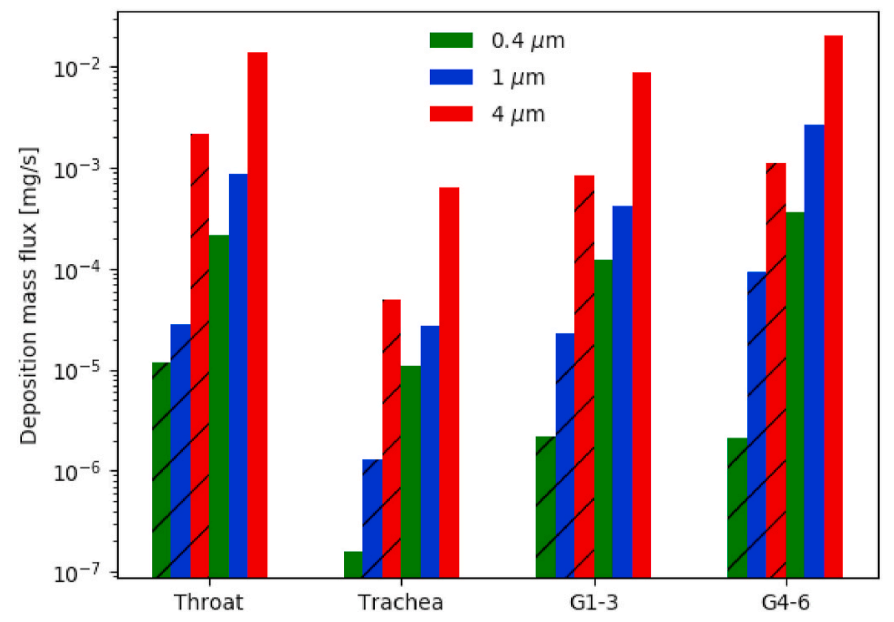

Fig. 8. Regional deposited mass flux for various inlet particle mass mean diameters $(0.4,1,4 \mu \mathrm{m})$ for evolving (solid filled bar) and non-evolving (hashed bar) aerosols of single species PG (simulation cases B-G in Table 3). 
results for the flow rate of $30 \mathrm{~L} / \mathrm{min}$ with the simulation results for the flow rate of $1.5 \mathrm{~L} / \mathrm{min}$ (Fig. 5), it can be observed that the aerosol equilibrium conditions are reached in deeper segments of the geometry for larger flow rates (consistent with the findings shown in Fig. 6).

Evaporation of particles influences the deposition fraction and reduces the aerosol mass delivered by individual particles depositing on the geometry surfaces. Fig. 10 shows the regional deposited mass for evolving and non-evolving aerosol simulations. A reduction in deposited mass because of evaporation is observed in all segments of the geometry and varies in the range of $50-70 \%$.

\subsubsection{Multispecies aerosol evolution and deposition under the influence of humidity and temperature}

We further extended our work from a single-species aerosol to a multispecies aerosol mixture in which the presence of water introduces the hygroscopic (condensation) effects in addition to the thermal effects discussed in the previous sections (case $\mathrm{J}$ in Table 3 ). Fig. 11 (a) shows the water condensation rate resulting in hygroscopic growth of the particles. Fig. 11 (b) shows the increase in the mass mean diameter of the particles due to hygroscopic and thermal effects. The maximum particle mass mean diameter is observed in the near wall region, where the humidity reaches maximum values. The figure shows that the condensational growth of particles is restricted to the throat region, similar to that observed in the single-species aerosol simulations presented in Section 3.2.1. The major condensation zones occur in proximity to the inlet region and also close to the throat upper wall regions, where the jet flow develops and impacts on the walls of the geometry.

For a multispecies evolving aerosol, we also investigated the changes in particles composition due to species-specific condensation and evaporation rates. In order to compare the species-specific evolution of the aerosol, Fig. 12 shows the fractions of each species in the liquid phase in the same thermal and humidity conditions as simulated and shown in Fig. 11. The distribution of species mass fractions in the liquid phase clearly shows the ability of different substances to evaporate. The highest value for the liquid mass fraction belongs to water, while the water fraction of the liquid at the inlet is of only $20 \%$. Fig. 11 (a) shows the water condensation rates. The larger condensation of water compared to other species can be explained by its higher vapor content in the system caused by the inhaled aerosol composition (Table 4) and the water saturation at the surface of the airways (Table 2). PG as the other semi-volatile species in the mixture condenses because of the changes in flow thermal conditions, and its liquid mass fraction increases. It can be observed in the same figure that VG has a uniform liquid mass fraction value, indicating that VG neither evaporates nor condenses, owing to its limited concentration in the gas phase under the simulated thermal conditions.

For better visualization of the aerosol evolution processes in the respiratory tract geometry, Fig. 13 shows the aerosol properties including the mass mean diameter and liquid/gas mass fractions of PG and water along four streamlines distributed in various branches of the geometry. These streamlines are generated by integrating the mixture velocity field and they do not strictly follow the path of the droplets but of the aerosol mixture. Some fluctuations in properties shown in the figure are expected as the streamlines are not necessarily in the center of the geometry, where conditions are more uniform, and they change their distance to the geometry walls, where we have implemented saturated boundary conditions. The results confirm major quantitative changes in mass mean diameter and mass fractions in two phases in the throat region.

Fig. 14 shows the regional deposition of a multispecies aerosol for non-evolving and evolving aerosol simulations. Fig. 14 (a) shows the deposition particle number density flux in various segments of the respiratory tract geometry for non-evolving and evolving aerosols simulated with the same thermal properties and flow conditions. Similarly to single-species aerosol simulations, there is a larger particle number deposition flux in the case of evolving aerosol. Fig. 14 (b) shows the regional deposition mass flux for each species, also comparing the results for non-evolving and evolving aerosols. The deposited masses of all species increase in case of the evolving aerosol, with the extent of increase being related to the volatility of the species.

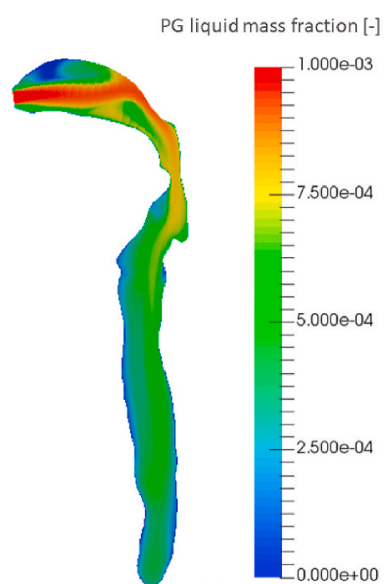

(a)

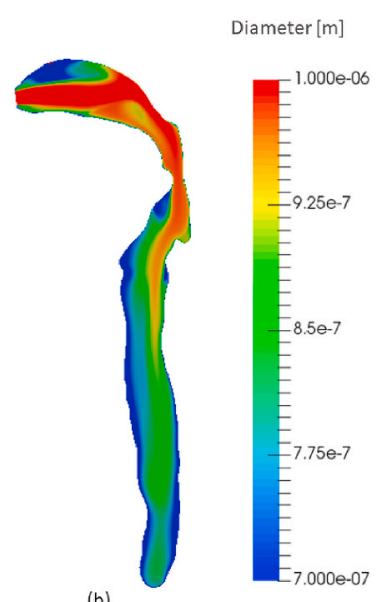

(b)

Fig. 9. (a) Snapshot of the liquid mass fraction of single-species PG aerosol with an inlet flow rate of $30 \mathrm{~L} / \mathrm{min}$. Aerosol at the inlet was simulated with temperature of $25^{\circ} \mathrm{C}$ and the cast temperature was constant at $37^{\circ} \mathrm{C}$. (b) Snapshot of the mass mean diameter of the particles flowing through the respiratory tract geometry. 


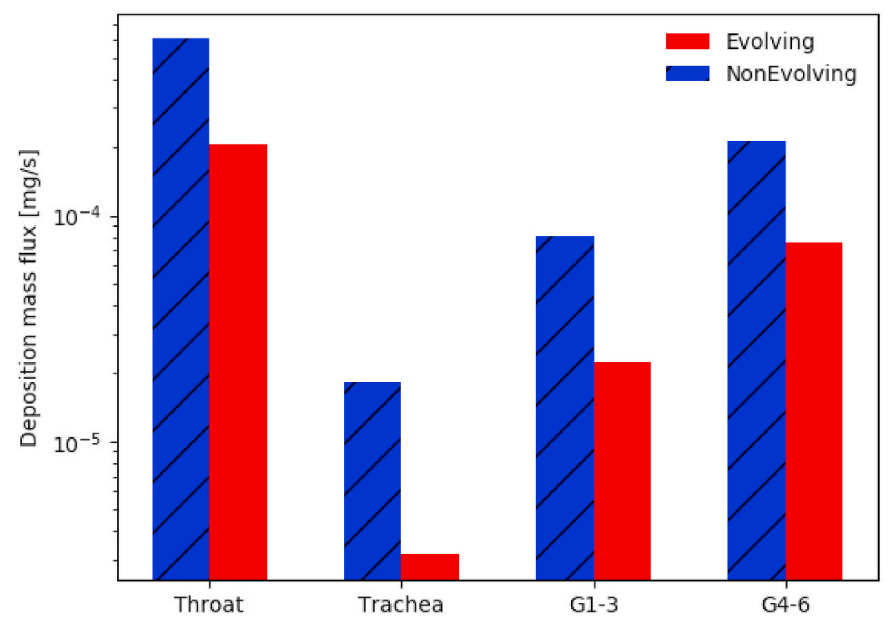

Fig. 10. Regional mass deposition flux compared for evolving and non-evolving single species PG aerosol simulations with an inhalation flow rate of $30 \mathrm{~L} / \mathrm{min}$ (simulation cases $\mathrm{H}$ and I in Table 3).
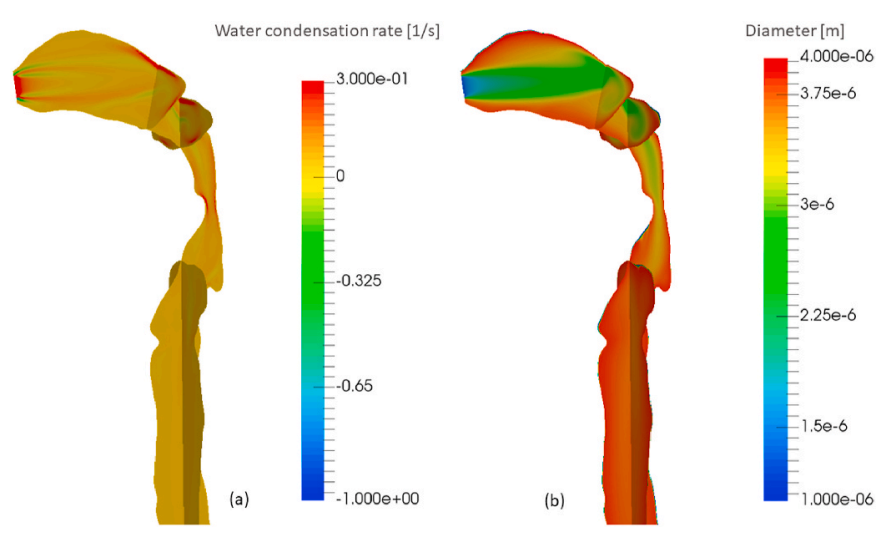

Fig. 11. (a) Snapshot of the water condensation rate for a multispecies aerosol with an inlet mixture of VG-PG-water with liquid mass fractions of $40-40-20 \%$ and corresponding saturated gas mass fractions at the inlet. The thermal conditions of flow change from $50^{\circ} \mathrm{C}$ at the inlet to $37^{\circ} \mathrm{C}$ at the wall.(b) Snapshot of the mass mean diameter of particles.

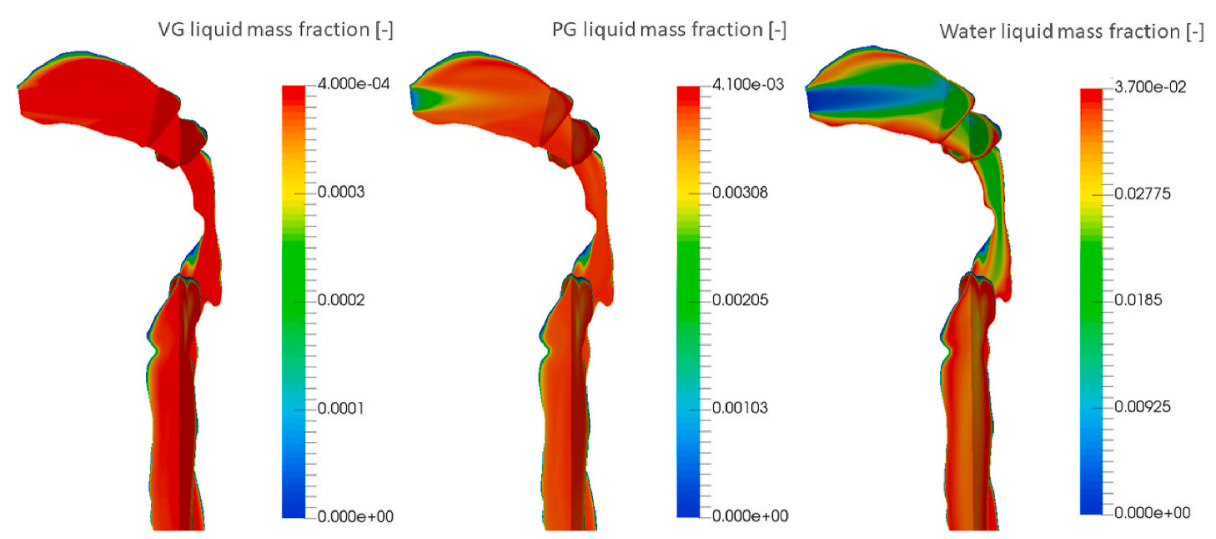

Fig. 12. Snapshots of the liquid mass fraction of each species of a multispecies aerosol with an inlet mixture of VG-PG-water with liquid mass fractions of $4 \cdot 10^{-4}, 4 \cdot 10^{-4}$, and $2 \cdot 10^{-4}$ for a steady inhalation flow rate of $1.5 \mathrm{~L} / \mathrm{min}$. 
(a)
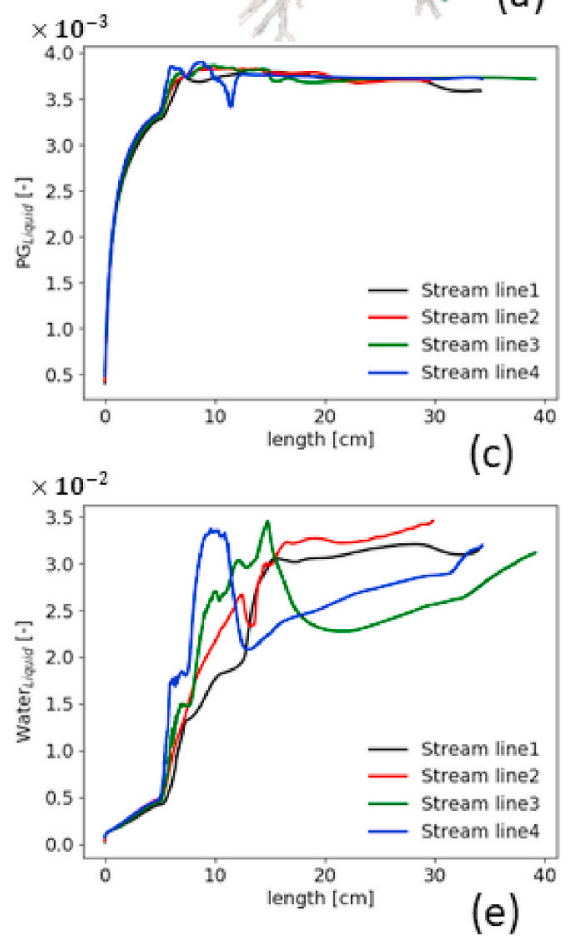
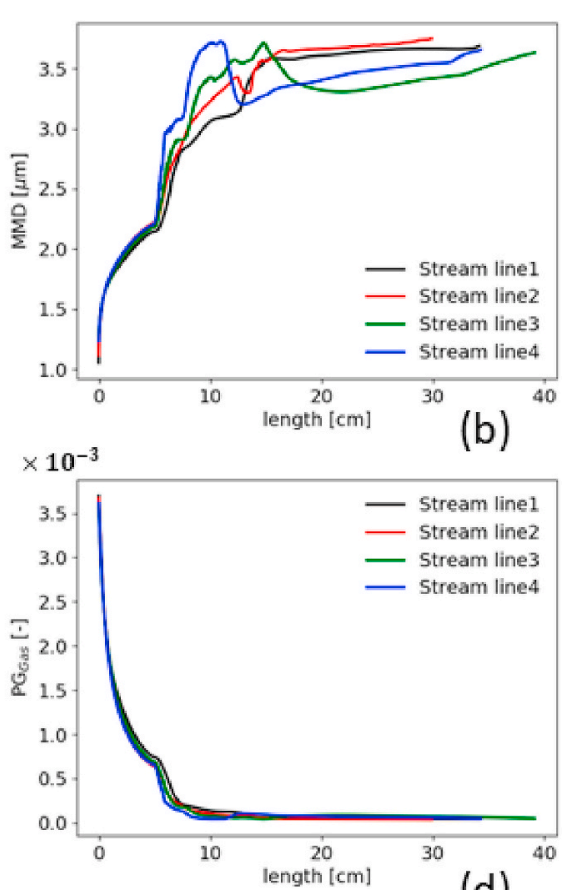

(d)

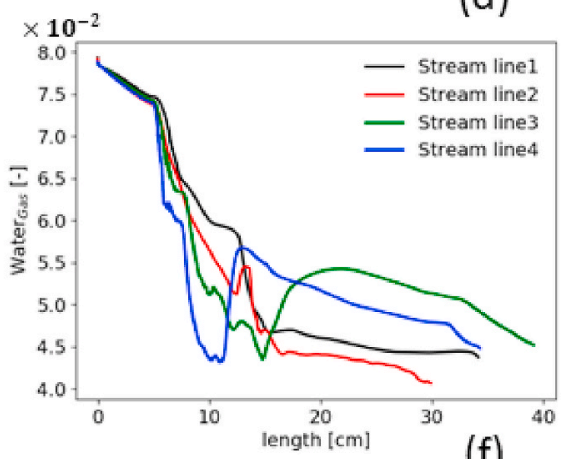

(f)

Fig. 13. (a) Four flow streamlines depicted in the respiratory tract geometry. (b) Particles mass mean diameter, (c) PG in the liquid phase, (d) PG in the gas phase, (e) water in the liquid phase and (f) water in the gas phase shown along the four streamlines.

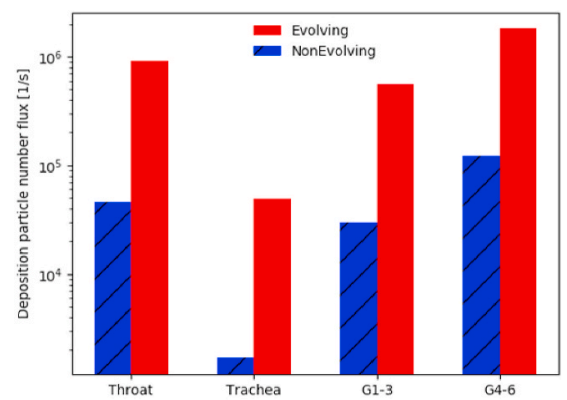

(a)

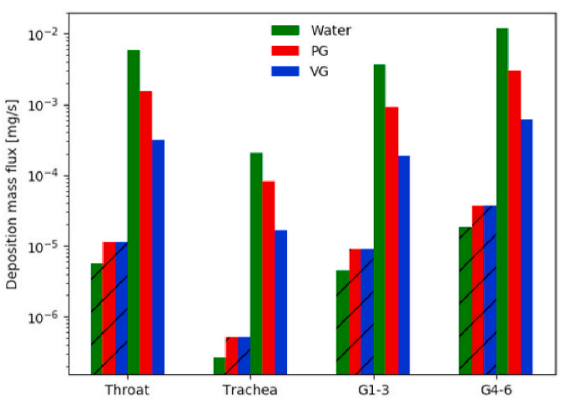

(b)

Fig. 14. (a) Particle number flux deposition in various segments of the cast geometry compared between non-evolving and evolving aerosol simulations with the same inlet mixture and thermal properties (simulation cases I and $\mathrm{J}$ in Table 3). (b) Regional mass deposition flux of each species compared for non-evolving (hashed bar) and evolving (solid filled bar) aerosol simulations. 
It is interesting to note that, despite the lack of VG mass transfer (owing to its limited volatility and presence in the gas phase), there is an increase in its deposition in the evolving scenario; this can be easily explained by the increased number of depositing particles containing VG. In other words, the high condensation rates of water and PG result in condensational growth of particles, leading to increased inertial deposition of particles that also contain the non-volatile VG.

\subsubsection{Evolution of nicotine in gas and liquid phases in a multispecies aerosol}

Finally, we present the most complex case of interest in which we simulated a multispecies mixture containing all species considered so far, along with volatile nicotine species with experimentally verified non-ideal vapor pressure behavior in the presence of water (See case L in Tables 3 and 4). Fig. 15 shows the relative mass fraction of nicotine in the gas phase divided by its mass fraction in the liquid phase. The figure shows that the chemical interactions of the species in the mixture determining the activity coefficient of individual species have a significant influence on evolution of the aerosol composition and partitioning of each species. The equilibrium conditions in aerosol evolution would consequently influence the final delivery of the aerosol in the human airways.

\section{Summary and concluding remarks}

In this study, we investigated aerosol evolution and deposition under the thermal and humidity conditions of the human respiratory tract. Computational simulations were performed by using an Eulerian simulation framework, AeroSolved (AeroSolved, 2019). A multispecies condensation/evaporation model was used to obtain species-specific condensation/evaporation rates by taking into account the non-ideal mixture behavior corrected by activity coefficients introduced to calculate vapor pressures at the surface of the liquid particles. For the simulated species, we used temperature dependent thermal properties, which allowed us to accurately resolve local gradients of the aerosol mixture properties due to temperature variations. The aerosol mixture at the inlet of the geometry was simulated with a saturated boundary conditions dependent on the inflow temperature. Warm and cold (with respect to the human body temperature) aerosols were simulated to obtain insights on aerosol evolution and deposition for various flows and aerosol conditions. We studied the phase mass transfer of aerosol particles at flow rates and conditions relevant for inhalation investigations. We found that temperature and humidity play very important roles in triggering aerosol evolution, changing phase partitioning of the species, and finally, influencing regional particles deposition as a highly particle size dependent process.

A multispecies evolving aerosol composed of VG, PG, and water was simulated to understand its species-specific evolution and deposition. The humidity conditions of the respiratory tract were also implemented, with $100 \%$ relative humidity at the walls of the geometry representing the water vapor released from the moist tissues of the respiratory tract. The combined effect of the temperature changes at the respiratory tract walls and the humidity conditions results in condensational growth or shrinkage of the particles. This leads to an increase or decrease in deposited mass on the geometry walls, depending on a multitude of factors, including aerosol equilibrium conditions, particles residence time in the airways, and particles deposition mechanisms. The extent of change in deposited mass varies for each species depending on its physico-chemical properties. Semi-volatile species such as PG and water undergo a great extent of evaporation/condensation, and their deposited masses change by up to two and three orders of magnitude, respectively. In contrast, VG does not evaporate/condense in the thermal conditions considered in this study. The deposited mass of VG in a multispecies aerosol is still subject to change, and the change in deposited mass is attributable to the increase in number of deposited particles driven by the increase in inertial deposition due to water and PG condensation. We must note the particular dynamics occurring along the developed flow and within the boundary layer in which the important processes have prolonged time scales for aerosol evolution.

There is currently no information regarding species interactions in large volumes of industrially relevant liquid mixtures; therefore, there are no experimental data or validated vapor-liquid equilibrium models available for such mixtures. With this in mind, we

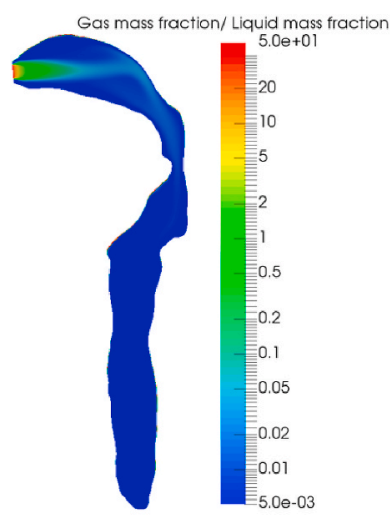

$\gamma=1$

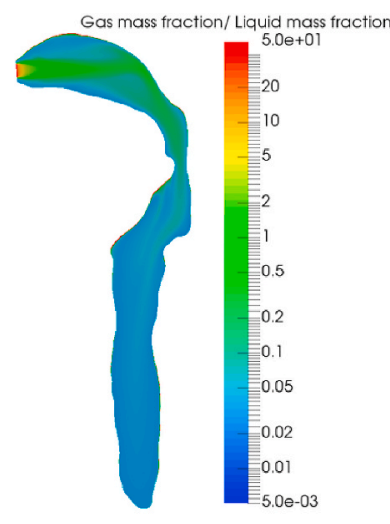

$\gamma=10$

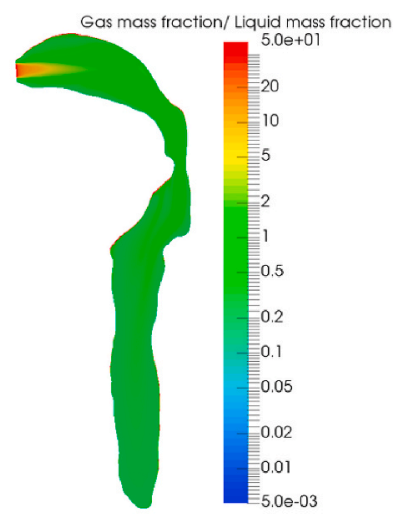

$\gamma=60$

Fig. 15. Relative mass fraction of nicotine in the gas phase divided by its mass fraction in the liquid phase shown for three choices of activity coefficient $(\gamma)$ equal to: 1,10 , and 60 . These simulations refer to case $\mathrm{L}$ in Table 3. 
performed sensitivity analysis by changing the activity coefficients of nicotine in the water, PG, VG mixture and compared the liquid/ gas mass fractions of nicotine when interacting in the aerosol mixture. This liquid composition resembles the typical liquid used in electronic cigarettes, which have recently gained a lot of attention from toxicological risk assessment perspective. The physicochemical properties of nicotine in the considered mixture are not yet well characterized. Increasing the activity coefficient of nicotine from 1 to 60 (with reference to experimentally determined nicotine activity coefficients in an aqueous solution (Banyasz, 1999)) causes a significant increase in vapor mass fraction during inhalation. This would allow nicotine to be absorbed better from the gas phase. It also indicates that the chemical composition of the aerosol plays an important role in the liquid gas partitioning of the species and, therefore, on its regional deposited mass in the human respiratory tract.

In our study, we omitted an aerosol coalescence model and instead focused on simulating aerosols with small particle number densities. For comparison between evolving and non-evolving simulations, we applied exactly the same inlet boundary conditions at equilibrium, which can be seen as a simulation assumption. We have also neglected vapor absorption on the respiratory walls and instead focused on liquid particle deposition and its variation under various inhalation conditions. We conducted simulations in a realistic geometry of the respiratory tract, including tracheobronchial branches up to six generations and; therefore, our conclusions are valid for the simulated geometry. Computational simulations can be conducted for more detailed geometries of the human lung depending on the availability of such geometries with future advances in medical imaging techniques. Our simulations have demonstrated the importance of accounting for appropriate condensation/evaporation modeling with the ability to deal with complex liquid mixtures. Further substantial efforts are needed to better validate these models with increasing role of aerosol chemistry, which is possible with our developed software capabilities.

\section{Disclosure}

All authors are employees of Philip Morris International. Philip Morris International is the sole source of funding and sponsor of this project.

\section{Declaration of competing interest}

The authors declare that they have no known competing financial interests or personal relationships that could have appeared to influence the work reported in this paper.

\section{Appendix A. Thermal properties of the mixture substances}

\section{Water in gas phase}

Molecular weight $(\mathrm{kg} /$ mole $)$

$$
M W=18.015
$$

Thermal conductivity $(\boldsymbol{J} /(\boldsymbol{m} . \boldsymbol{s} . K))$

$$
k(T)=6.2041 \cdot 10^{-6} T^{1.3973}
$$

Dynamic viscosity (Pa.s)

$$
\mu(T)=1.7096 \cdot 10^{-8} T^{1.1146}
$$

Heat capacity at constant pressure $(J / K)$

$$
C_{p}(T)=1851.9567+1487.0941(2610.5 / \sinh (2610.5 / T))^{2}+493.81071(1169 / \cosh (1169 / T))^{2}
$$

Saturated vapor pressure $P_{s}(\mathrm{~Pa})$

$$
P_{s}(T)=e^{73.649-7258.2 / T-7.3037 \ln (T)+4.1653 \cdot 10^{-6} T^{2}}
$$

\section{Water in liquid phase}

$$
\begin{aligned}
& \text { Density }\left(\mathrm{kg} / \mathrm{m}^{3}\right) \\
& \qquad \rho(T)=3.280712 \cdot 10^{-5} T^{3}-0.03440865 T^{2}+11.53645 T-249.5258
\end{aligned}
$$

Thermal conductivity $(J /(m . s . K))$

$$
k(T)=1.861 \cdot 10^{-9} T^{3}-8.078 T^{2}+0.0057255 T-0.432
$$

Dynamic viscosity (Pa.s) 


$$
\mu(T)=e^{-52.843+3703.6 / T+5.866 \ln (T)-5.879 T^{10}}
$$

Heat capacity at constant pressure $(J / K)$

$$
C_{p}(T)=5.20127671 \cdot 10^{-7} T^{4}-0.000783569248 T^{3}+0.451013045 T^{2}-116.019983 T+15341.1046
$$

\section{Glycerol in gas phase}

Molecular weight $(\mathrm{kg} / \mathrm{mole})$

$$
M W=92.09
$$

Thermal conductivity $(J /(m . s . K))$

$$
k(T)=2.026 \cdot 10^{-8} T^{2}+5.9 \cdot 10^{-5} T-0.009158
$$

Dynamic viscosity (Pa.s)

$$
\mu(T)=3.28 \cdot 10^{-13} T^{2}+2.2666 \cdot 10^{-8} T-1.46 \cdot 10^{-8}
$$

Heat capacity at constant pressure $(J / K)$

$$
\begin{aligned}
& C_{p}\left(T, T_{a}\right)=90.2862\left(16.2564+\left((-11.1044-16.2564)\left(T_{a}\right)^{2}\right)(1.0-(830.0781 /(830.0781+\right. \\
& \left.\left.T))\left(-25.4696+162.1117 T_{a}-309.8217 T_{a}^{2}+212.4448 T_{a}^{3}\right)\right)\right), \\
& T_{a}=T /(830.0781+T)
\end{aligned}
$$

Saturated vapor pressure $(P a)$

$$
\begin{aligned}
& P_{s}\left(T, T_{r}\right)=7500000 e^{850 / T\left(-6.94758 T_{r}-0.33345 T_{r}^{1.5}-5.98569 T_{r}^{2.5}-1.33011 T_{r}^{5.0}\right)}, \\
& T_{r}=1.0-T / 850
\end{aligned}
$$

\section{Glycerol in liquid phase}

Density $\left(\mathrm{kg} / \mathrm{m}^{3}\right)$

$$
\begin{aligned}
& \rho\left(T, T_{r}\right)=349+1341.5932 T_{r}^{0.35}-1168.205 T_{r}^{2 / 3}+1429.7634 T_{r}-527.771 T_{r}^{4 / 3}, \\
& T_{r}=1.0-T / 850
\end{aligned}
$$

Thermal conductivity $(J /($ m.s.K $))$

$$
k(T)=1.02 \cdot 10^{-13} T^{4}-1.05 \cdot 10^{-10} T^{3}+2.3 \cdot 10^{-8} T^{2}+0.000119 T+0.2562
$$

Dynamic viscosity (Pa.s)

$$
\begin{aligned}
& \mu\left(T, T_{b}\right)=0.0079964 e^{T_{b}^{1 / 3}\left(-3.91153+6.54946 T_{b}\right)}, \\
& T_{b}=(582.48-T) /(T-73.885)
\end{aligned}
$$

Heat capacity at constant pressure $(J / K)$

$$
\begin{aligned}
& C_{p}\left(T, T_{r}\right)=8.314462\left(58.5772-49.1381 T_{r}\right) / 0.09209, \\
& T_{r}=1.0-T / 850
\end{aligned}
$$

\section{Propylene glycol in gas phase}

Molecular weight $(\mathrm{kg} /$ mole $)$

$$
M W=76.094
$$

Thermal conductivity $(J /(m . s . K))$ 


$$
k(T)=0.0001666 T^{0.9765} /(1.0+706 / T)
$$

Dynamic viscosity (Pa.s)

$$
\mu(T)=4.543 \cdot 10^{-8} T^{0.9173} /(1.0+61 / T)
$$

Heat capacity at constant pressure $(J / K)$

$$
C_{p}(T)=2643.3096+1062.1074(1865.6 / \sinh (1865.6 / T))^{2}-3207.086(279.98 / \cosh (279.98 / T))^{2}
$$

Saturated vapor pressure $(P a)$

$$
P_{s}(T)=e^{212.8-15420 / T-28.109 \ln (T)+2.1564 \cdot 10^{-5} T^{2}}
$$

\section{Propylene glycol in liquid phase}

Density $\left(\mathrm{kg} / \mathrm{m}^{3}\right)$

$$
\rho(T)=83.11748 / 0.26106^{1.0+(1.0-T / 626)^{0.20459}}
$$

Thermal conductivity $(\boldsymbol{J} /(\boldsymbol{m} . \boldsymbol{s} . K))$

$$
k(T)=-4.97 \cdot 10^{-5} T+0.2152
$$

Dynamic viscosity (Pa.s)

$$
\mu(T)=e^{-804.54+30487 / T+130.79 \ln (T)-0.15449 T}
$$

Heat capacity at constant pressure $(J / K)$

$$
C_{p}(T)=763.2665 T+5.850658
$$

\section{Nicotine in gas phase}

Molecular weight $(\mathrm{kg} /$ mole $)$

$$
M=162
$$

Thermal conductivity $(J /(m . s . K))$

$$
k(T)=0.15105
$$

Dynamic viscosity (Pa.s)

$$
\mu(T)=4.543 \cdot 10^{-8} T^{0.9173} /(1.0+61 / T)
$$

Heat capacity at constant pressure $(J / K)$

$$
\begin{aligned}
& C_{p}\left(T, T_{a}\right)=50.14\left(18.91+\left((-9.93-18.91)\left(T_{a}\right)^{2}\right)\left(1.0-(764.09 /(764.09+T))\left(-31.52+172.42 T_{a}-303.98 T_{a}^{2}+193.87 T_{a}^{3}\right)\right)\right), \\
& T_{a}=T /(764.09+T)
\end{aligned}
$$

Saturated vapor pressure $(\mathrm{Pa})$

$$
P_{s}(T)=0.0021673 / T
$$

\section{Nicotine in liquid phase}

Density $\left(\mathrm{kg} / \mathrm{m}^{3}\right)$

$$
\rho(T)=127.49 / 0.3213^{1.0+(1.0-T / 742.9)^{0.38}}
$$

Thermal conductivity $(\boldsymbol{J} /($ m.s.K $))$

$$
k(T)=-0.003521\left(1-T_{r}\right)^{\left(2.3 e^{-5}+9.35 e^{-8} T_{r}\right)}
$$


$T_{r}=1.0-T / 742.9$

Dynamic viscosity (Pa.s)

$\mu(T)=e^{12.09+331.88 / T-0.1 \ln (T)-2.08 T^{0.38}}$

Heat capacity at constant pressure $(J / K)$

$C_{p}(T)=1758.45$

\section{References}

AeroSolved. (2019) Accessed December 12 https://www.aerosolved.com.

Asgari, M., Lucci, F., Bialek, J., Dunan, B., Andreatta, G., Smajda, R., Lani, S., Blondiaux, N., Majeed, S., Steiner, S., et al. (2019). Development of a realistic human respiratory tract cast representing physiological thermal conditions. Aerosol Science and Technology, $10,1-11$.

Asgari, M., Lucci, F., \& Kuczaj, A. (2019b). Multispecies aerosol evolution and deposition in a bent pipe. Journal of Aerosol Science, 129, 53-70.

Banyasz, J. (1999). The physical chemistry of nicotine. Amsterdam: Elsevier.

Dolovich, M. (2001). Measuring total and regional lung deposition using inhaled radiotracers. Journal of Aerosol Medicine, 14(1), 35-44.

Fleming, J., Halson, P., Conway, J., Moore, E., Nassim, M., Hashish, A., Bailey, A., Holgate, S., \& Martonen, T. (1996). Three-dimensional description of pulmonary deposition of inhaled aerosol using data from multimodality imaging. Journal of Nuclear Medicine, 37(5), 873-877.

Frederix, E., Kuczaj, A., Nordlund, M., Belka, M., Lizal, F., Jedelskỳ, J., Elcner, J., Jícha, M., \& Geurts, B. (2018). Simulation of size-dependent aerosol deposition in a realistic model of the upper human airways. Journal of Aerosol Science, 115, 29-45.

Frederix, E., Kuczaj, A., Nordlund, M., Veldman, A., \& Geurts, B. (2017). Eulerian modeling of inertial and diffusional aerosol deposition in bent pipes. Computers \& Fluids, 159, 217-231.

Frederix, E., Stanic, M., Kuczaj, A., Nordlund, M., \& Geurts, B. (2016). Characteristics-based sectional modeling of aerosol nucleation and condensation. Journal of Computational Physics, 326, 499-515.

Grgic, B., Finlay, W., Burnell, P., \& Heenan, A. (2004). In vitro intersubject and intrasubject deposition measurements in realistic mouth-throat geometries. Journal of Aerosol Science, 35(8), 1025-1040.

Heyder, J., Gebhart, J., Rudolf, G., Schiller, C., \& Stahlhofen, W. (1986). Deposition of particles in the human respiratory tract in the size range 0.005-15 $\mu$ m. Journal of Aerosol Science, 17(5), 811-825.

Hinds, W. (2012). Aerosol technology: Properties, behavior, and measurement of airborne particles. John Wiley \& Sons.

Lee, K., \& Chen, H. (1984). Coagulation rate of polydisperse particles. Aerosol Science and Technology, 3, 327-334.

Longest, P., \& Hindle, M. (2010). CFD simulations of enhanced condensational growth (ecg) applied to respiratory drug delivery with comparisons to in vitro data. Journal of Aerosol Science, 41(8), 805-820.

Longest, P., \& Vinchurkar, S. (2007). Validating CFD predictions of respiratory aerosol deposition: Effects of upstream transition and turbulence. Journal of Biomechanics, 40(2), 305-316.

Longest, P. W., \& Xi, J. (2007). Computational investigation of particle inertia effects on submicron aerosol deposition in the respiratory tract. Journal of Aerosol Science, 38(1), 111-130.

Newbold, F., \& Amundson, N. (1973). A model for evaporation of a multicomponent droplet. American Institute of Chemical Engineers Journal, 19(1), 22-30.

Nordlund, M., Belka, M., Kuczaj, A., Lizal, F., Jedelsky, J., Elcner, J., Jicha, M., Sauser, Y., Le Bouhellec, S., Cosandey, S., et al. (2017). Multicomponent aerosol particle deposition in a realistic cast of the human upper respiratory tract. Inhalation Toxicology, 29(3), $113-125$.

Park, S., \& Wexler, A. (2008). Size-dependent deposition of particles in the human lung at steady-state breathing. Journal of Aerosol Science, 39(3), 266-276.

Stahlhofen, W., Gebhart, J., \& Heyder, J. (1980). Experimental determination of the regional deposition of aerosol particles in the human respiratory tract. American Industrial Hygiene Association Journal, 41(6), 385-398a.

Tu, H., \& Ray, A. (2005). Measurement of activity coefficients from unsteady state evaporation and growth of microdroplets. Chemical Engineering Communications, 192 (4), 474-498.

Van Leer, B. (1979). Towards the ultimate conservative difference scheme. V. A second-order sequel to Godunov's method. Journal of Computational Physics, 32(1), 101-136.

Winkelmann, C., Nordlund, M., Kuczaj, A., Stolz, S., \& Geurts, B. (2014). Efficient second-order time-integration for single-species aerosol formation and evolution. International Journal for Numerical Methods in Fluids, 74(5), 313-334.

Zhang, Z., Kleinstreuer, C., Donohue, J., \& Kim, C. (2005). Comparison of micro-and nano-size particle depositions in a human upper airway model. Journal of Aerosol Science, 36(2), 211-233.

Zhang, Z., Kleinstreuer, C., \& Hyun, S. (2012). Size-change and deposition of conventional and composite cigarette smoke particles during inhalation in a subjectspecific airway model. Journal of Aerosol Science, 46, 34-52. 